Research Article

\title{
Mean Shift-Based Mobile Localization Method in Mixed LOS/NLOS Environments for Wireless Sensor Network
}

\author{
Xiaosheng Yu, Peng Ji, Ying Wang, and Hao Chu \\ Faculty of Robot Science and Engineering, Northeastern University, Shenyang 110819, China \\ Correspondence should be addressed to Xiaosheng Yu; yuxiaosheng7@163.com
}

Received 28 March 2017; Accepted 26 October 2017; Published 25 December 2017

Academic Editor: Jaime Lloret

Copyright ( 2017 Xiaosheng Yu et al. This is an open access article distributed under the Creative Commons Attribution License, which permits unrestricted use, distribution, and reproduction in any medium, provided the original work is properly cited.

\begin{abstract}
Mobile localization estimation is a significant research topic in the fields of wireless sensor network (WSN), which is of concern greatly in the past decades. Non-line-of-sight (NLOS) propagation seriously decreases the positioning accuracy if it is not considered when the mobile localization algorithm is designed. NLOS propagation has been a serious challenge. This paper presents a novel mobile localization method in order to overcome the effects of NLOS errors by utilizing the mean shift-based Kalman filter. The binary hypothesis is firstly carried out to detect the measurements which contain the NLOS errors. For NLOS propagation condition, mean shift algorithm is utilized to evaluate the means of the NLOS measurements and the data association method is proposed to mitigate the NLOS errors. Simulation results show that the proposed method can provide higher location accuracy in comparison with some traditional methods.
\end{abstract}

\section{Introduction}

Wireless localization is one of the technologies in the fields of the intelligent robot, national security, and health surveillance [1] and has received the researcher's considerable attention in the past decades. With the increase of the demand for positioning service, many wireless positioning systems have been developed, among of which Global Positioning System (GPS) is one of the most popular localization systems. However, GPS is not able to provide the desirable performance when the receiver is in indoor environments. Wireless sensor network (WSN) is a novel technology with rapid diffusion. Location is a significant application of WSN. The WSN-based location methods have widely been used for indoor location $[2,3]$.

In the WSN-based localization approaches' design, the location of the beacon nodes and the measurements between the beacon nodes and unknown node are assumed to be the known prior information. Generally, there are four measurement methods: time of arrival (TOA) [4], time difference of arrival (TDOA) [5], received signal strength (RSS) [6], and angle of arrival (AOA) [7]. However, there are many objects located in some practical environments. These objects may block the direct propagation path which leads to the non- line-of-sight (NLOS) environments. The measurement contains a positive bias which is termed as NLOS error. In this environment, the performance of the conventional positioning methods will degrade dramatically. Therefore, the accurate localization in the NLOS environments has been a significant topic.

In this paper, we propose a novel location algorithm which can solve the NLOS errors. This paper is structured as follows: related works are introduced in Section 2. System model and mean shift methods are described in Section 3. Section 4 presents the proposed algorithm. The performance evolution of the proposed algorithm is shown in Section 5 . Section 6 presents the conclusions.

\section{Related Works}

In order to solve the NLOS errors, researchers proposed numerous methods. These methods can be generally divided into two types [8]: hard-decision ones and soft-decision ones. In the first methods, an identification and discard strategy is employed, which means that the NLOS measurements are firstly identified and then discarded. The localization is only dependent on the line-of-sight (LOS) measurements. There are many promising approaches to be proposed to estimate 
the propagation paths [8-11]. These kinds of algorithms require accurate identification and enough LOS measurements which are not suitable in some practical environments. The second methods utilize all of the measurements with different weights to locate the target. The interacting multiple model (IMM) with different filter approaches such as the Kalman filer [12], the extended Kalman filer [6, 13, 14], the cubature Kalman filer [15], and the hidden Markov models [16] can be considered as the most classical soft-decision methods. These kinds of methods are practical when only a small number of measurements can be used for positioning. Most of the methods mentioned above were designed with the prior information of the NLOS errors. But, in practical and complicated environments, the prior information is usually unknown.

There are many approaches proposed to realize the accurate location without any prior knowledge of statistical information of the NLOS measurements. These methods are termed as nonparametric methods. In [17], Chen proposed a residual weighting (Rwgh) algorithm. The residuals are presented to compute the weights for the initial node coordinates from the least squares estimation of all possible combinations of the measurements. The final localization result is obtained by weighting these initial results. In [18], $\mathrm{Yu}$ et al. constructed a voting matrix to estimate the initial localization results and then employed the residual weighting to acquire the final estimated position. In [19, 20], Garcia et al. utilized a training strategy to obtain the training measurements. The final position is estimated by using these measurements. In [21], Lloret et al. proposed a novel stochastic algorithm which is based on a combination of deductive and inductive methods to decide the estimated position. In $[22,23]$, the statistical features including mean, variance, and Rician $\mathrm{K}$ factor are used to train the support vector machine (SVM) classifier to identify the propagation condition. They can obtain the desirable results with enough training samples. In [24], a min-max strategy is invented to identify the propagation paths by constructing a detection region according to the range measurements. If the localization is far away from the edge of the region, the propagation is an NLOS propagation. In [25], Cheng et al. employed the Gaussian mixture distributions to describe the distributions of NLOS errors and the estimated mean is used for poisoning in the Kalman filter frame. In [26], Hu et al. proposed a probabilistic data-association-based IMM approach to improve the location accuracy in the rough environment. The position estimation is performed by using an IMM frame and then the PDA approach is employed to correct it.

Most of the nonparametric methods mentioned above were designed with the assumption that the obstacles are fixed. But, in the practical and complicated environments, the positions of the obstacles may be changed dynamically. These nonparametric methods cannot provide desirable position estimation in mixed LOS/NLOS environments where some obstacles are always moving. This paper presents an efficient mobile node localization approach which is termed as improved Kalman filter (IKF) based on mean shift [27] to overcome the NLOS effect in mixed LOS/NLOS environments. Our algorithm has the desirable location ability
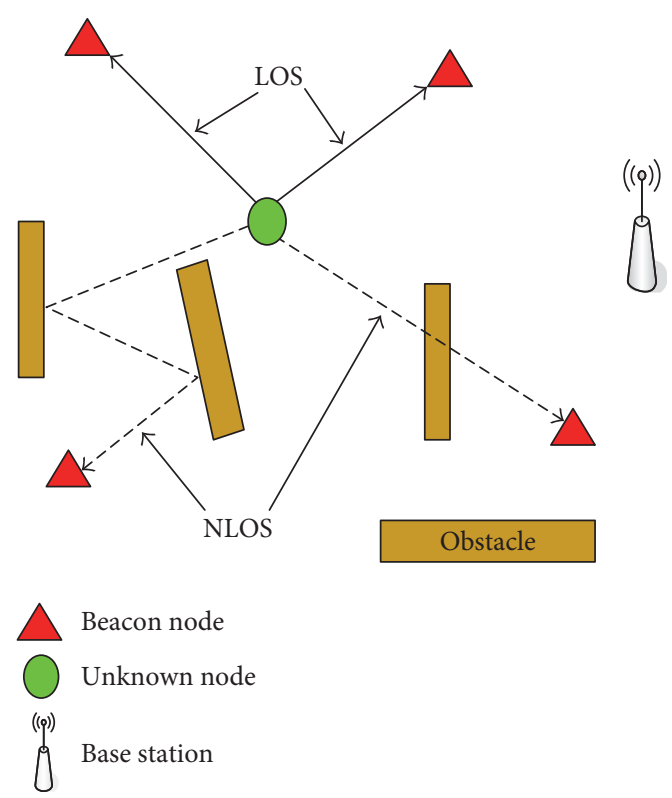

FIgURE 1: The LOS/NLOS propagation.

without any prior knowledge of statistical information of the NLOS measurements. The simulation results demonstrate its effectiveness.

\section{Background}

In this section, we consider the scenario with $M$ beacon nodes and an unknown node. These beacon nodes are randomly distributed, and their coordinates are known which are given by $\theta_{n}=\left(x_{n}, y_{n}\right)^{T}$, where $n=(1, \ldots, N)$. There are many obstacles deployed in the field whose positions are not given. At time $k$, the target is moving in the area and its position is denoted by $(x(k), y(k)) k=(1,2, \ldots K)$. The wireless signal is transmitted from the beacon nodes to the unknown node. The measured distance is estimated by TOA. The illustration of the LOS/NLOS propagation is show in Figure 1.

3.1. System Model. At time $k$, the range measurements between the unknown node and $n$th $(n=1, \ldots, N)$ beacon node can be acquired, which is represented by the following:

$$
z_{n}(k)=d_{n}(k)+\varepsilon_{n}, \quad k=1, \ldots, K,
$$

where $d_{n}(k)=\sqrt{\left(x(k)-x_{n}\right)^{2}+\left(y(k)-y_{n}\right)^{2}}$ stands for the true distance. $\varepsilon_{n}$ is noise which has different forms in the LOS environment and NLOS environment. Generally, $\varepsilon_{n}$ is modeled by

$$
\varepsilon_{n}= \begin{cases}v_{n}, & \text { LOS; } \\ v_{n}+b_{\mathrm{NLOS}}, & \text { NLOS, }\end{cases}
$$

where $v_{n}$ is the measurement noise, $v_{n} \sim N\left(0, \sigma_{n}^{2}\right)$. The NLOS error $b_{\text {NLOS }}$ is often regarded as a positive bias due to the longer indirect propagation path in NLOS condition and independent of $v_{n}$. The NLOS error $b_{\mathrm{NLOS}}$ is different from 
$v_{n}$ which may follow different distributions [28], such as Gaussian distribution, uniform distribution, and exponential distribution in different conditions.

In the LOS propagation environment, the probability density function (PDF) of $\varepsilon_{n}$ is

$$
p^{L}\left(\varepsilon_{n}\right)=\frac{1}{\sqrt{2 \pi \sigma_{n}^{2}}} \exp \left(-\frac{\varepsilon_{n}^{2}}{2 \sigma_{n}^{2}}\right) .
$$

In the NLOS propagation environment, $\varepsilon_{n}$ has different forms because the NLOS error $b_{\text {NLOS }}$ may obey different distributions. When $b_{\text {NLOS }}$ obeys a Gaussian distribution, $b_{\mathrm{NLOS}} \sim N\left(\mu_{b}, \sigma_{b}^{2}\right)$, the PDF of $\varepsilon_{n}$ is

$$
p^{\mathrm{NL}}\left(\varepsilon_{n}\right)=\frac{1}{\sqrt{2 \pi \sigma_{\varepsilon}^{2}}} \exp \left(-\frac{\left(\varepsilon_{n}-\mu_{b}\right)^{2}}{2 \sigma_{e}^{2}}\right)
$$

where $\sigma_{\varepsilon}^{2}=\sigma_{b}^{2}+\sigma_{n}^{2}$. When $b_{\text {NLOS }}$ obeys a uniform distribution, $b_{\mathrm{NLOS}} \sim U\left(u_{\min }, u_{\max }\right)$, the PDF of $\varepsilon_{n}$ can be described as follows:

$$
p^{\mathrm{NL}}\left(\varepsilon_{n}\right)=\frac{1}{u_{\max }-u_{\min }}\left[Q\left(\frac{\varepsilon_{n}-u_{\max }}{\sigma_{n}}\right)-Q\left(\frac{\varepsilon_{n}-u_{\min }}{\sigma_{n}}\right)\right],
$$

where $Q()$ stands for the cumulative distribution function of the standard normal distribution. When $b_{\mathrm{NLOS}}$ obeys an exponential distribution, $b_{\mathrm{NLOS}} \sim E(\lambda)$, the PDF of $\varepsilon_{n}$ is as follows:

$$
p^{\mathrm{NL}}\left(\varepsilon_{n}\right)=\frac{\lambda}{2} \exp \left(-\lambda\left(\varepsilon_{n}-\frac{\lambda^{2} \sigma_{n}^{2}}{2}\right)\right) \Phi\left(\frac{\lambda \sigma_{n}^{2}-\varepsilon_{n}}{\sqrt{2} \sigma_{n}}\right),
$$

where $\lambda$ is a positive constant and $\Phi$ is the complementary error function.

3.2. Mean Shift Method. The prior knowledge of the NLOS errors cannot be obtained in practical environment. The mean shift method is employed to approximate the probability density. It is assumed that there are $M$ range measurements $\widehat{\mathbf{z}}(k)=\left[\widehat{\mathbf{z}}^{1}(k), \ldots, \widehat{\mathbf{z}}^{M}(k)\right]$ at time $k$. For an initial estimate $z(k)$, the weighted mean of the measurements $\widehat{\mathbf{z}}(k)$ is determined as follows:

$$
v(z(k))=\frac{\sum_{\widehat{\mathbf{z}}^{j}(k) \in N(z(k))} K^{M}\left(\widehat{\mathbf{z}}^{j}(k)-z(k)\right) \widehat{\mathbf{z}}^{j}(k)}{\sum_{\widehat{\mathbf{z}}^{j}(k) \in N(z(k))} K^{M}\left(\widehat{\mathbf{z}}^{j}(k)-z(k)\right)},
$$

where $N(z(k))$ stands for the neighborhood of the initial estimate $z(k)$ and $N(z(k)) \in \widehat{\mathbf{z}}(k)$ and $K^{M}(x) \neq 0$ stands for the kernel function with the following forms:

$$
\begin{aligned}
& K_{E}^{M}(x)= \begin{cases}c\left(1-\|x\|^{2}\right), & \|x\| \leq 1, \\
0, & \text { otherwise, }\end{cases} \\
& K_{U}^{M}(x)= \begin{cases}c & \|x\| \leq 1, \\
0, & \text { otherwise, }\end{cases} \\
& K_{N}^{M}(x)=c \cdot \exp \left(-\frac{1}{2}\|x\|^{2}\right) .
\end{aligned}
$$

This kernel function is used to determine the weights of the neighborhood data to re-estimate the mean. In the practical application, there are many initial estimates required to obtain the desirable results. The weighted means can be obtained through an iteration process. This method always sets the initial estimates $z(k)$ constantly to obtain the new estimations of $v(z(k))$, and the estimation process always repeats until $v(z(k))$ is converged.

\section{Proposed NLOS Localization Method}

The range measurements play the significant roles in the whole process of mobile localization. We adopt the highfrequency measurements [29], the reliable data. Therefore, at time $k$, we can obtain $M$ range measurements $\widehat{\mathbf{z}}_{n}(k)=$ $\left[\widehat{\mathbf{z}}_{n}^{1}(k), \ldots, \widehat{\mathbf{z}}_{n}^{M}(k)\right]$ between the target and the $n$th beacon node. Their mean value is

$$
\gamma_{n}(k)=\frac{1}{M} \sum_{m=1}^{M} \widehat{\mathbf{z}}_{n}^{m}(k) .
$$

We define the following state vector of the unknown node relative to the $n$th beacon node at time $k$ :

$$
\mathbf{X}_{n}(k)=\left[\gamma_{n}(k), \dot{\gamma}_{n}(k)\right]^{T}, \quad k=1, \ldots, K,
$$

where $\dot{\gamma}_{n}(k)$ stands for the velocity.

The corresponding state model is

$$
\mathbf{X}_{n}(k+1)=\mathbf{A} \mathbf{X}_{n}(k)+\mathbf{G} \omega_{n}(k)
$$

where $\mathbf{A}=\left[\begin{array}{ll}1 & t \\ 0 & 1\end{array}\right]$ stands for the state transition matrix, $\mathbf{G}=\left[\begin{array}{c}t^{2} / 2 \\ t\end{array}\right], t$ stands for the sample period, and $\omega_{n}(k)$ stands for the random process noise.

The measurement equation in the mixed propagation environment is

$$
Z_{n}(k)=\mathbf{H X}_{n}(k)+\varepsilon_{n}
$$

where $\mathbf{H}=[1,0]$ stands for the observation matrix and $\varepsilon_{n}$ is the noise.

Figure 2 indicates the architecture of the proposed improved Kalman filter. Firstly, the Kalman prediction step is carried out. Secondly, a hypothesis and an alternative method are utilized to detect the channel conditions. Thirdly, in the NLOS condition, the mean shift method is used to calculate the weighted means of the range measurements and a novel algorithm is proposed to provide the measurement residual for data association. Fourthly, the Kalman update step is implemented. Finally, the maximum likelihood algorithm is employed to obtain the final position with the filtered range measurements. The detailed steps of the proposed algorithm are listed below.

Step 1 (Kalman predication). It is assumed that $\widehat{\mathbf{X}}_{n}(0 \mid 0) \sim N$ $\left(X_{n}(0), \mathbf{P}_{n}(0 \mid 0)\right)$ is known to complete the initialization of the Kalman filter. For the time interval $k=1, \ldots, K$, 


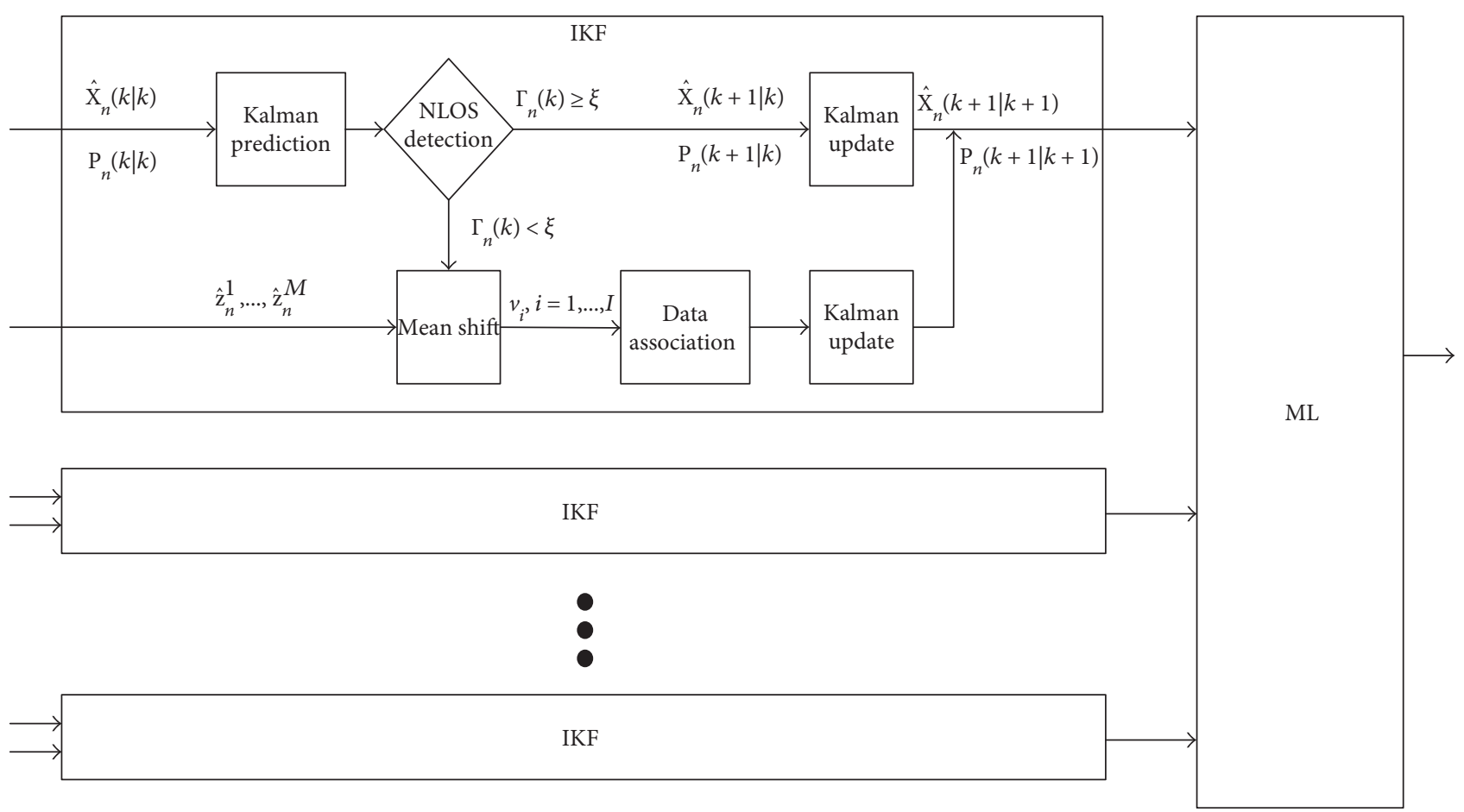

FIgURE 2: Structure of the improved Kalman filter.

the conventional time update equation of Kalman filter is expressed as follows:

$$
\begin{aligned}
& \widehat{\mathbf{X}}_{n}(k+1 \mid k)=\mathbf{A} \widehat{\mathbf{X}}_{n}(k \mid k), \\
& \mathbf{P}_{n}(k+1 \mid k)=\mathbf{A} \mathbf{P}_{n}(k \mid k) \mathbf{A}^{T}+\mathbf{G Q G}^{T},
\end{aligned}
$$

where $\widehat{\mathbf{X}}_{n}(k+1 \mid k)$ and $\widehat{\mathbf{X}}_{n}(k \mid k)$ represent the predicted and updated state estimate, respectively, of the state vector of the unknown node relative to the $n$th beacon at time $k . \mathbf{P}_{n}(k \mid k)$ and $\mathbf{P}_{n}(k+1 \mid k)$ represent the predicted and updated covariance. $\mathbf{Q}$ denotes the variance of the process noise.

The measurement residual is defined by the following:

$$
\begin{aligned}
E_{n}(k+1) & =\gamma_{n}(k+1)-\widehat{Z}_{n}(k+1 \mid k), \\
\widehat{\mathbf{Z}}_{n}(k+1 \mid k) & =\mathbf{H} \widehat{\mathbf{X}}_{n}(k+1 \mid k) .
\end{aligned}
$$

The innovation covariance matrix is expressed as follows:

$$
S_{n}(k+1)=\mathbf{H} \mathbf{P}_{n}(k+1 \mid k) \mathbf{H}^{T}+\mathbf{Q} .
$$

The Kalman gain is expressed as follows:

$$
\mathbf{K}_{n}(k+1)=\mathbf{P}_{n}(k+1 \mid k) \mathbf{H}^{T}\left(S_{n}(k+1)\right)^{-1} .
$$

Step 2 (NLOS detection). We employ the hypotheses and alternatives [8] to detect the NLOS propagation. According to the above equations, in LOS condition, it can be summarized that

$$
E_{n}(k) \sim N\left(0, S_{n}(k)\right)
$$

where $N\left(0, S_{n}(k)\right)$ stands for the Gaussian density function of $E_{n}(k)$ with zero mean and variance $S_{n}^{2}(k)$. Due to (18), the test statistic $\Gamma_{n}(k)$ is defined as follows:

$$
\Gamma_{n}(k)=\left(E_{n}(k)\right)^{T} S_{n}(k) E_{n}(k) .
$$

The following hypotheses and alternative are utilized to identify the propagation condition:

$$
\begin{aligned}
& H_{0}: \Gamma_{n}(k) \geq \xi, \\
& H_{1}: \Gamma_{n}(k)<\xi,
\end{aligned}
$$

where $\xi$ is the threshold. If $\Gamma_{n}(k)$ is larger than $\xi$, the hypotheses, $H_{0}$, and the range measurements are obtained in the LOS condition. Otherwise, the range measurements contain the numerous NLOS errors.

Step 3 (mean shift-based data association). In the NLOS condition, the mean shift method is employed to compute the weighted means of the measurements $\widehat{\mathbf{z}}_{n}(k)$ with the corresponding $l$ initial estimates $z(k)=\left[z^{1}(k), \ldots, z^{l}(k)\right]$.

$$
v_{i}(z(k))=\frac{\sum_{\widehat{\mathbf{z}}^{j}(k) \in N(z(k))} K^{M}\left(\widehat{\mathbf{z}}^{j}(k)-z(k)\right) \widehat{\mathbf{z}}^{j}(k)}{\sum_{\widehat{\mathbf{z}}^{j}(k) \in N(z(k))} K^{M}\left(\widehat{\mathbf{z}}^{j}(k)-z(k)\right)} .
$$

The output result $v_{i}$ can be obtained through an iterative process, and the corresponding measurement residuals are given by the following:

$$
E_{i}(k+1)=v_{i}-\widehat{\mathbf{Z}}_{n}(k+1 \mid k), \quad i=1, \ldots, I .
$$


If $v_{i}$ is the LOS measurement, it is similar to the predicted measurement. Hence, the weights for each measurement residual are given by the following:

$$
\mu_{i}(k)=\frac{N\left(E_{i}(k) ; \quad 0, S_{n}(k)\right)}{\sum_{i=1}^{I} N\left(E_{i}(k) ; \quad 0, S_{n}(k)\right)} .
$$

The output of the mean shift-based data association is expressed as

$$
E_{n}(k)=\sum_{i=1}^{I} E_{i}(k) \mu_{i}(k)
$$

Step 4 (Kalman update). In the LOS environment, $E_{n}(k)$ is computed according to (14). In the NLOS environment, $E_{n}(k)$ is computed according to (24). The final state estimate can be obtained using the following equation:

$$
\widehat{\mathbf{X}}_{n}(k+1 \mid k+1)=\widehat{\mathbf{X}}_{n}(k+1 \mid k)+\mathbf{K}_{n}(k+1) E_{n}(k+1) .
$$

The covariance can be updated as follows:

$\mathbf{P}_{n}(k+1 \mid k+1)=\mathbf{P}_{n}(k+1 \mid k)-\mathbf{K}_{n}(k+1) S_{n}(k+1)\left(\mathbf{K}_{n}(k+1)\right)^{T}$.

After obtaining the state estimation vector $\widehat{\mathbf{X}}_{n}(k+1 \mid k+1)$, the filtered measurements are as follows:

$$
\tilde{\mathbf{z}}_{n}(k)=\Omega \widehat{\mathbf{X}}_{n}(k+1 \mid k+1), \quad \Omega=[1,0] .
$$

Step 5 (ML-based location). We use the ML method to realize the final localization estimation. As mentioned above, the coordinates of the beacon nodes are $\left(x_{1}, y_{1}\right),\left(x_{2}, y_{2}\right), \ldots$, $\left(x_{n}, y_{n}\right)$ as the prior information. The evaluated coordinate of the target is denoted by $\mathbf{Y}(k)=[x(k), y(k)]^{T}$, and the processed range measurements are denoted by $\tilde{\mathbf{z}}_{n}(k)$ at time $k$. The following linear equation is summarized as follows:

$$
\mathbf{D Y}(k)=\mathbf{B},
$$

where

$$
\begin{aligned}
& \mathbf{D}=2\left[\begin{array}{cc}
\left(x_{1}-x_{2}\right) & \left(y_{1}-y_{2}\right) \\
\left(x_{1}-x_{3}\right) & \left(y_{1}-y_{3}\right) \\
\vdots & \vdots \\
\left(x_{1}-x_{N}\right) & \left(y_{1}-y_{N}\right)
\end{array}\right], \\
& \mathbf{B}=\left[\begin{array}{c}
{\left[\tilde{\mathbf{z}}_{2}(k)\right]^{2}-\left[\tilde{\mathbf{z}}_{1}(k)\right]^{2}-\left(x_{1}^{2}+y_{1}^{2}\right)+\left(x_{2}^{2}+y_{2}^{2}\right)} \\
{\left[\tilde{\mathbf{z}}_{3}(k)\right]^{2}-\left[\tilde{\mathbf{z}}_{1}(k)\right]^{2}-\left(x_{1}^{2}+y_{1}^{2}\right)+\left(x_{3}^{2}+y_{3}^{2}\right)} \\
\vdots \\
{\left[\tilde{\mathbf{z}}_{N}(k)\right]^{2}-\left[\tilde{\mathbf{z}}_{1}(k)\right]^{2}-\left(x_{1}^{2}+y_{1}^{2}\right)+\left(x_{N}^{2}+y_{N}^{2}\right)}
\end{array}\right] .
\end{aligned}
$$

The final position of the moving target can be obtained as follows:

$$
\mathbf{Y}(k)=\left(\mathbf{D}^{\mathrm{T}} \mathbf{D}\right)^{-1} \mathbf{D}^{\mathrm{T}} \mathbf{B}
$$

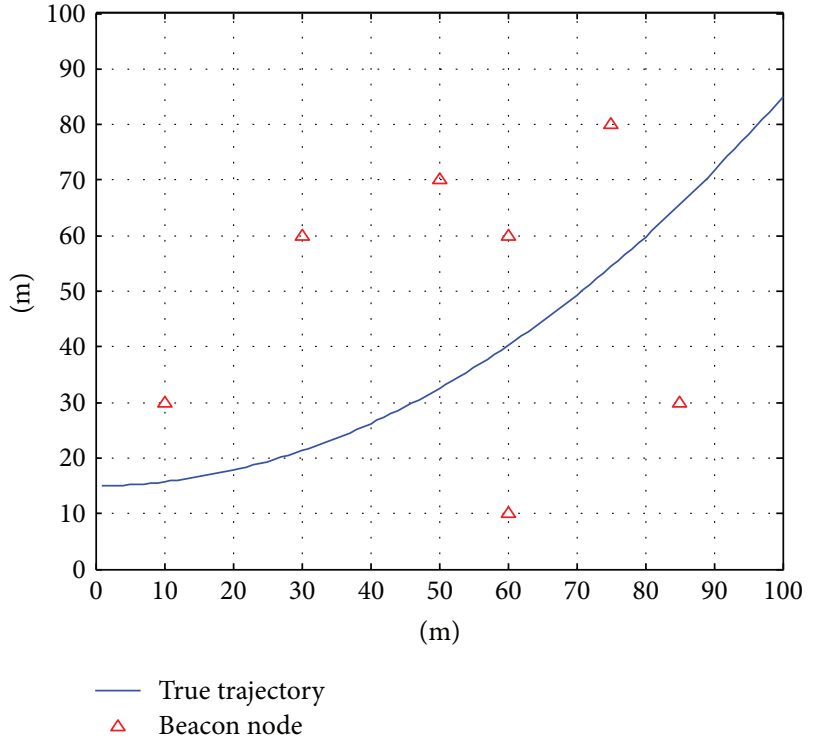

Figure 3: Diagram of the simulation environment.

\section{Performance Evaluation}

The location ability of the proposed approach in the mixed LOS/NLOS environments is tested through the following simulations in this section. The proposed improved Kalman filter (IKF) algorithm is compared with the maximum likelihood (ML) algorithm, the residual weighting (Rwgh) algorithm, and the Kalman filter (KF) algorithm to validate its effectiveness. We consider a $100 \mathrm{~m} \times 100 \mathrm{~m}$ square area. There are seven beacon nodes in this area. The target is moving in this field with the velocity of $1 \mathrm{~m} / \mathrm{s}$. The obstacles are distributed randomly, and their positions are always changed dynamically. Figure 3 shows the diagram of the simulation environment. We assumed that the communication ranges for all sensor nodes are the same which are equal to $150 \mathrm{~m}$. The measure noise $v_{n}$ is the white Gaussian noise with variance $\sigma_{n}$ (defaulted as 1 ), $v_{n} \sim N(0,1)$. The NLOS errors obey Gaussian distribution, uniform distribution, and exponential distribution, respectively. We carry out 2000 Monte Carlo runs to obtain the simulation results of these four algorithms in each case.

The location ability of these four approaches is evaluated by the average location error:

$$
\mathrm{ALE}=\frac{1}{K \cdot t_{i}} \sum_{i=1}^{t_{i}} \sum_{k=1}^{K} \sqrt{\left(x(k)-\widehat{\mathbf{x}}_{i}(k)\right)^{2}+\left(y(k)-\widehat{\mathbf{y}}_{i}(k)\right)^{2}},
$$

where $K=2000, t_{i}=100$, and $[x(k), y(k)]$ and $\left[\widehat{\mathbf{x}}_{i}(k), \widehat{\mathbf{y}}_{i}(k)\right]$ denote the real position and the estimated position of the moving target at time $k$.

Firstly, we discuss the location ability of these four methods in the case of Gaussian distribution, in which the NOLS error $b_{\mathrm{NLOS}} \sim N\left(\mu_{b}, \sigma_{b}^{2}\right)$. Both Figures 4 and 5 display the different simulation results in the two cases. The relationship between the mean of the NLOS errors $\mu_{b}$ and the average location error is indicated in Figure 4. During the whole 


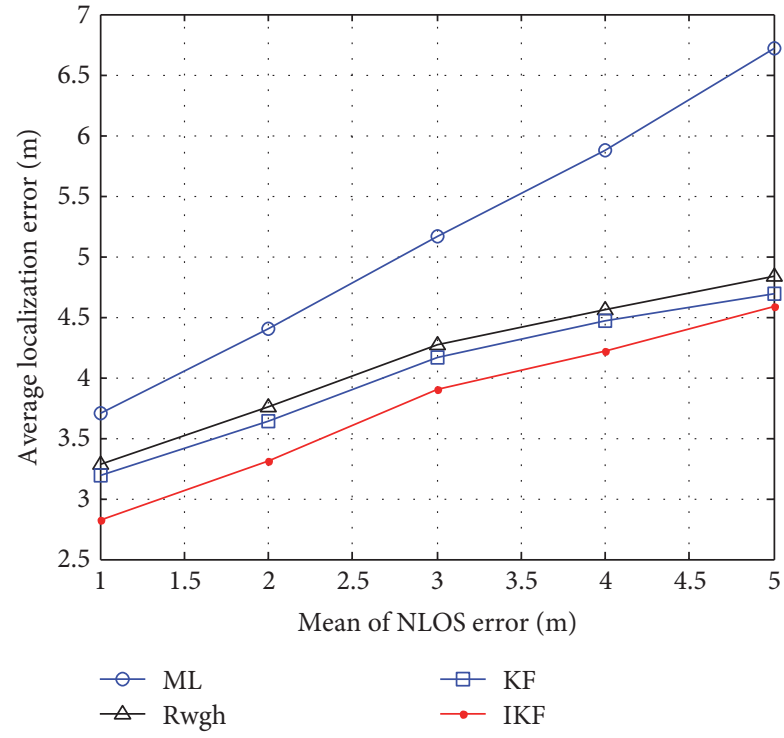

Figure $4: \mu_{b}$ versus ALE.

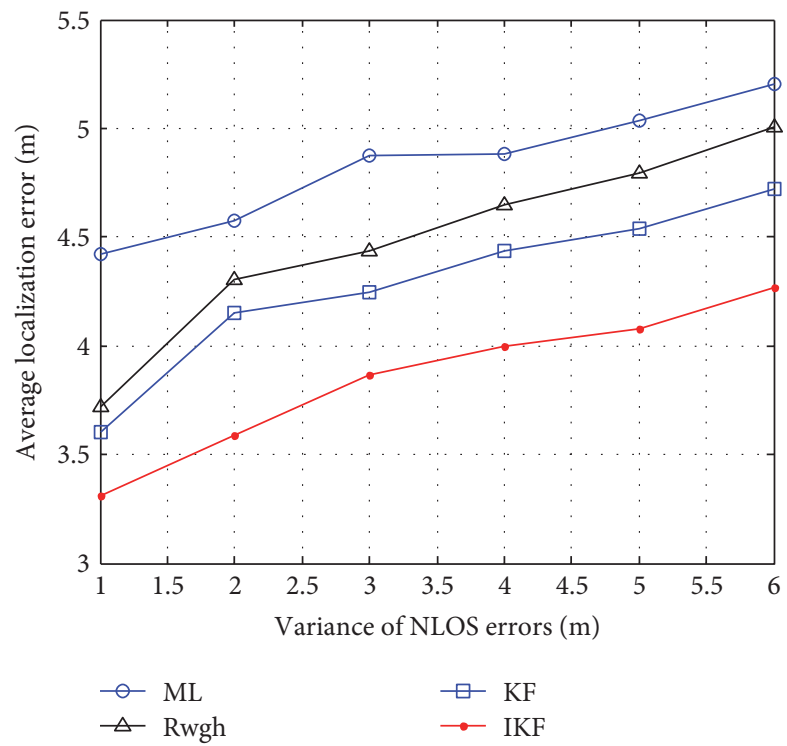

Figure 5: $\sigma_{b}^{2}$ versus ALE.

simulation process, the variance of the NLOS errors $\sigma_{b}^{2}$ is equal to 3. The mean of NLOS errors varies from 1 to 5 . Obviously, the average location errors of these four algorithms all rise when the parameter $\mu_{b}$ increases. The localization ability of ML is the worst because its average location errors have the most rapid rising. The proposed IKF method outperforms the other three methods. It always has the highest localization accuracy than ML, Rwgh, and KF methods, about $26.73 \%, 14.17 \%$, and $13.69 \%$, respectively.

We illustrate the variance of NLOS errors versus the average location error of these four approaches as shown in Figure 5. In this simulation, the parameter $\mu_{b}$ is equal to 3 . The variance of NLOS errors varies from 1 to 6 . It can be seen that these four algorithms are all sensitive to the variance of

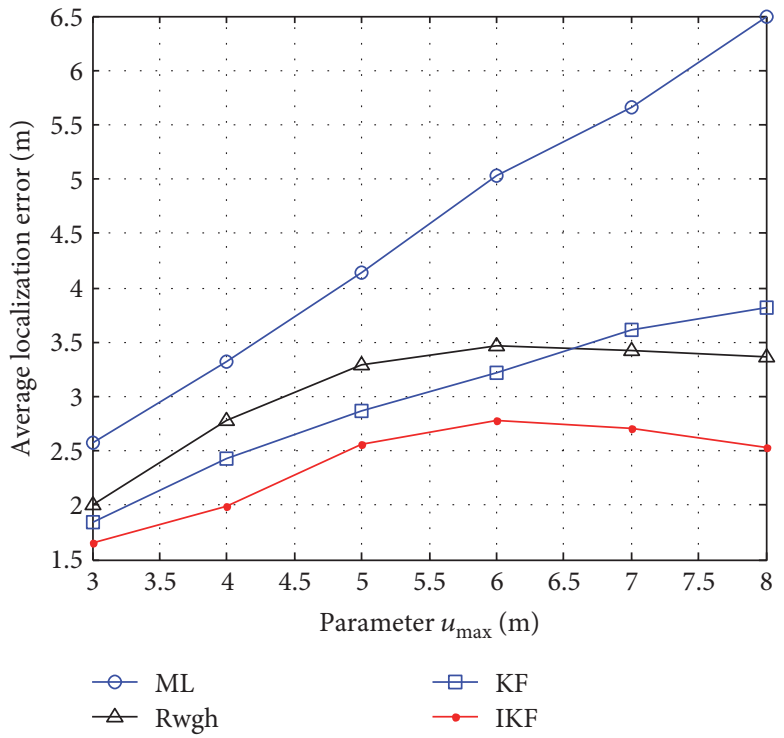

Figure 6: $u_{\max }$ versus ALE.

NLOS errors. The location performance degrades with the increment of the variance of NLOS errors. By contrast, the proposed IKF method always has the best performance than the other three methods.

Secondly, we discuss the location ability of these four methods in the case of uniform distribution, in which the NOLS error $b_{\mathrm{NLOS}} \sim U\left(u_{\min }, u_{\max }\right)$. In this simulation, the parameter $u_{\min }$ is equal to 3 . Figure 6 indicates the relationship between the parameter $u_{\max }$ and the average location error. It is obvious that the performance of the ML methods is almost the worst. The average location error of the KF method always rises with the increment of the parameter $u_{\max }$. The average location errors of the both the Rwgh and IKF algorithms rise when the parameter $u_{\max } \leq 6$. The location accuracy of both the Rwgh and IKF algorithms improves significantly when $u_{\max }>6$ due to their robustness to the large NLOS errors. The performance of the IKF algorithm is always the best.

Finally, we investigate the performance of the four approaches with the assumption that the NLOS errors obey the exponential distribution $b_{\mathrm{NLOS}} \sim E(\lambda)$. In this simulation, the parameter $\lambda$ varies from 1 to 6 . Figure 7 indicates the relationship between the parameter $\lambda$ and the average location error. It is obvious that the ML method always owns the worst location performance. The proposed method offers the better location estimation than the other three methods.

\section{Conclusion}

We investigated the mobile localization in rough environments and presented a novel IKF algorithm which can realize the accurate mobile node localization. The proposed IKF algorithm is independent of prior information. In the whole location process, the NLOS errors are completely unknown. In the simulation, the proposed method is compared with three traditional algorithms. The simulation results illustrate that the proposed IKF approach has the best performance. It 


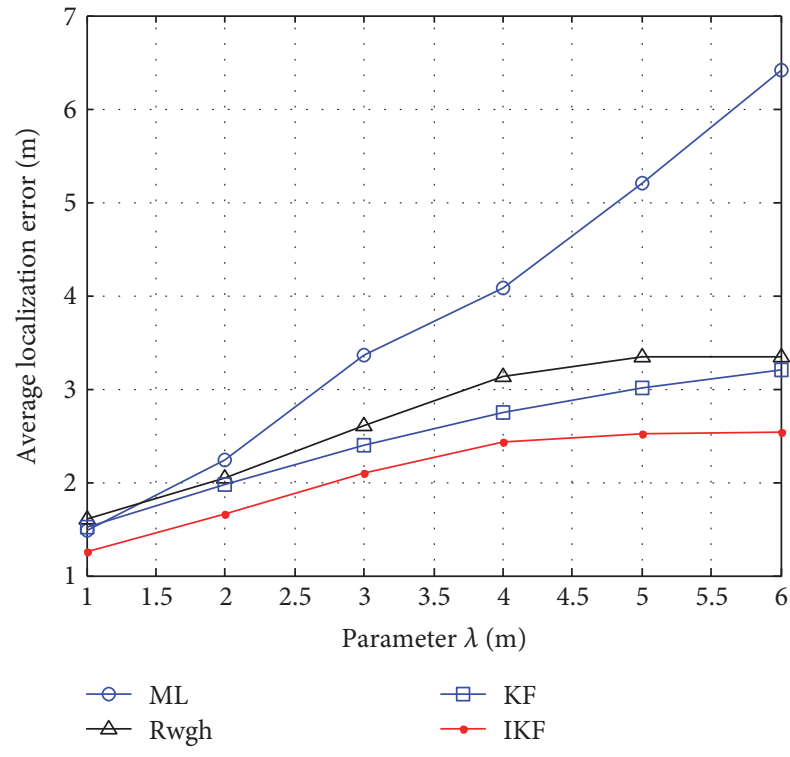

Figure 7: $\lambda$ versus ALE.

has higher localization accuracy than KF, Rwgh, and ML methods about $32.8 \%, 17.19 \%$, and $13.07 \%$, respectively. In the future, we will focus on the robust localization method with the mobile beacon nodes in the mixed LOS/NLOS environments.

\section{Conflicts of Interest}

The authors declare that there is no conflict of interests regarding the publication of this article.

\section{Acknowledgments}

This work was supported in part by the National Natural Science Foundation of China under Grant nos. 61701101, 61603080, 61603415, and 61503274 and the Fundamental Research Fund for the Central Universities of China (N162610004, N160404003, and N150503009).

\section{References}

[1] C. H. Ou, "A localization scheme for wireless sensor networks using mobile anchors with directional antennas," IEEE Sensors Journal, vol. 11, no. 7, pp. 1607-1616, 2011.

[2] Y. Liu, S. Zhang, and Y. Jing, "Non-line of sight node localization algorithm based on particle swarm optimization for wireless sensor networks," Control and Decision, vol. 30, no. 6, pp. 1106-1110, 2015.

[3] W. Cui, C. Wu, W. Meng, and L. Xie, "Dynamic multidimensional scaling algorithm for 3-D mobile localization," IEEE Transactions on Instrumentation and Measurement, vol. 65, no. 12, pp. 2853-2865, 2016.

[4] I. Guvenc and C. C. Chong, "A survey on TOA based wireless localization and NLOS mitigation techniques," IEEE Communications Surveys \& Tutorials, vol. 11, no. 3, pp. 107-124, 2009.

[5] Q. Liang, B. Zhang, and C. Zhao, "TDoA for passive localization: underwater versus terrestrial environment," IEEE
Transactions on Parallel and Distributed Systems, vol. 24, no. 10, pp. 2100-2108, 2013.

[6] B. Chen, C. Yang, F. Liao, and J. Liao, "Mobile location estimator in a rough wireless environment using extended Kalmanbased IMM and data fusion," IEEE Transactions on Vehicular Technology, vol. 58, no. 3, pp. 1157-1169, 2009.

[7] L. Cong and W. Zhuang, "Nonline-of-sight error mitigation in mobile location," IEEE Transactions on Wireless Communications, vol. 4, no. 2, pp. 560-573, 2005.

[8] U. Hammes and A. M. Zoubir, "Robust mobile terminal tracking in NLOS environments based on data association," IEEE Transactions on Signal Processing, vol. 58, no. 11, pp. 5872-5882, 2010.

[9] L. Cheng, C. Wu, Y. Zhang, and Y. Wang, "An indoor localization strategy for a mini-UAV in the presence of obstacles," International Journal of Advanced Robotic Systems, vol. 9, no. 4, pp. 153-158, 2012.

[10] S. Venkatesh and R. M. Buehrer, "Non-line-of-sight identification in ultra-wideband systems based on received signal statistics," IET Microwaves, Antennas \& Propagation, vol. 1, no. 6, pp. 1120-1130, 2007.

[11] N. Hu, C. Wu, P. Liu, H. Wu, B. Wu, and L. Cheng, "Vote selection mechanisms and probabilistic data associationbased mobile node localization algorithm in mixed LOS/NLOS environments," Telecommunication Systems, vol. 62, no. 4, pp. 641-655, 2016.

[12] J. Liao and B. Chen, "Robust mobile location estimator with NLOS mitigation using interacting multiple model algorithm," IEEE Transactions on Wireless Communications, vol. 5, no. 10, pp. 3002-3006, 2006.

[13] U. Hammes and A. M. Zoubir, "Robust MT tracking based on M-estimation and interacting multiple model algorithm," IEEE Transactions on Signal Processing, vol. 59, no. 7, pp. 3398-3409, 2011.

[14] Y. Zhang, W. Fu, S. Xiang, D. Wei, and B. Yang, "IMM-EKF algorithm-based indoor moving target localization," Journal of Computer Research and Development, vol. 51, no. 11, pp. 2408-2415, 2015.

[15] W. Li and Y. Jia, "Location of Mobile station with maneuvers using an IMM-based cubature Kalman filter," IEEE Transactions on Industrial Electronics, vol. 59, no. 11, pp. 4338-4348, 2012.

[16] J. Ru, C. Wu, Z. Jia, Y. Yang, Y. Zhang, and N. Hu, “An indoor mobile location estimator in mixed line of sight/non-line of sight environments using replacement modified hidden Markov models and an interacting multiple model," Sensors, vol. 15, no. 6, pp. 14298-14327, 2015.

[17] P. C. Chen, "A non-line-of-sight error mitigation algorithm in location estimation," in WCNC. 1999 IEEE Wireless Communications and Networking Conference (Cat. No.99TH8466), pp. 316-320, New Orleans, LA, USA, September 1999.

[18] X. Yu, C. Wu, and L. Cheng, "Indoor localization algorithm for TDOA measurement in NLOS environments," IEICE Transactions on Fundamentals of Electronics Communications and Computer Sciences, vol. E97A, no. 5, pp. 11491152, 2014.

[19] M. Garcia, C. Martinez, J. Tomas, and J. Lloret, "Wireless Sensors Self-Location in an Indoor WLAN Environment," in 2007. SensorComm 2007. International Conference on Sensor Technologies and Applications, pp. 14-20, Valencia, Spain, October 2007. 
[20] M. Garcia, J. Tomas, F. Boronat, and J. Lloret, "The Development of Two Systems for Indoor Wireless Sensors Self-Location," Ad Hoc \& Sensor Wireless Networks, vol. 8, no. 3-4, pp. 235-258, 2009.

[21] J. Lloret, J. Tomas, M. Garcia, and A. Canovas, "A hybrid stochastic approach for self-location of wireless sensors in indoor environments," Sensors, vol. 9, no. 5, pp. 3695-3712, 2009.

[22] Z. Xiao, H. Wen, A. Markham, N. Trigoni, P. Blunsom, and J. Frolik, "Non-line-of-sight identification and mitigation using received signal strength," IEEE Transactions on Wireless Communications, vol. 14, no. 3, pp. 1689-1702, 2015.

[23] S. Marano, W. M. Gifford, and H. Wymeersch, "NLOS identification and mitigation for localization based on UWB experimental data," IEEE Journal on Selected Areas in Communications, vol. 28, no. 7, pp. 1026-1035, 2010.

[24] S. Kong, Y. Kwon, and G. Park, "Robust localization over obstructed interferences for inbuilding wireless applications," IEEE Transaction on Consumer Electronics, vol. 55, no. 1, pp. 499-518, 2009.

[25] L. Cheng, Y. Wang, H. Wu, N. Hu, and C. Wu, "Non-parametric location estimation in rough wireless environments for wireless sensor network," Sensors and Actuators, vol. 224, pp. 57-64, 2015.

[26] N. Hu, H. Wu, C. Wu, and Y. Gao, "A novel mobile localization method for distributed sensor network with non-line-ofsight error mitigation," International Journal of Distributed Sensor Networks, vol. 10, no. 4, article 154896, 2014.

[27] D. Comaniciu and P. Meer, "Mean shift: a robust approach toward feature space analysis," IEEE Transactions on Parallel and Distributed Systems, vol. 24, no. 5, pp. 603-619, 2002.

[28] X. Shi, G. Mao, Z. Yang, and J. Chen, "Localization algorithm design and performance analysis in probabilistic LOS/NLOS environment," in 2016 IEEE International Conference on Communications (ICC), pp. 1-6, Kuala Lumpur, Malaysia, May 2016.

[29] K. Yu and E. Dutkiewicz, "NLOS identification and mitigation for mobile tracking," IEEE Transactions on Aerospace and Electronic Systems, vol. 49, no. 3, pp. 1438-1452, 2013. 


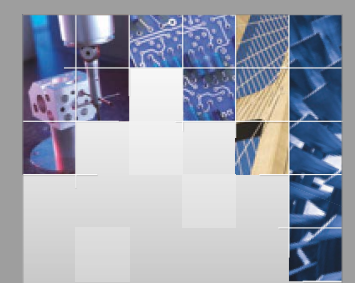

\section{Enfincering}
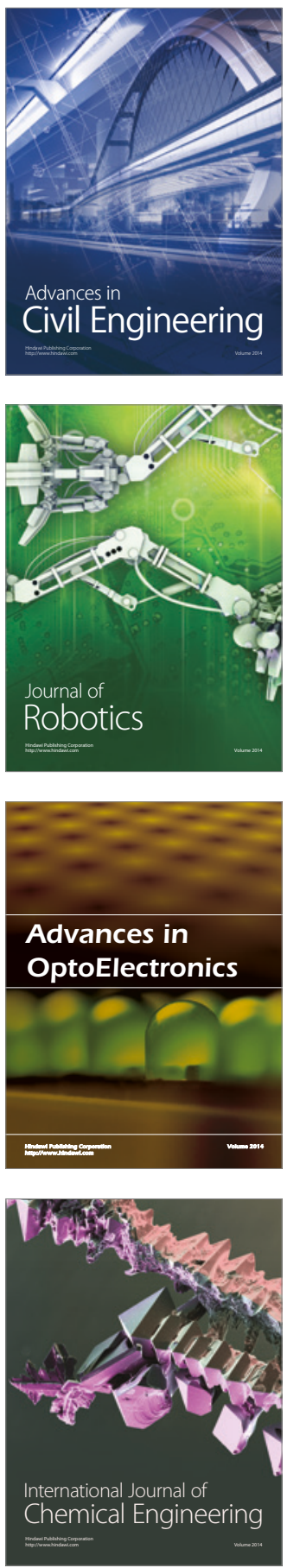

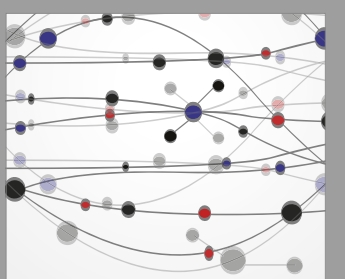

The Scientific World Journal

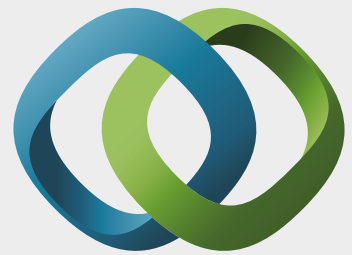

\section{Hindawi}

Submit your manuscripts at

https://www.hindawi.com
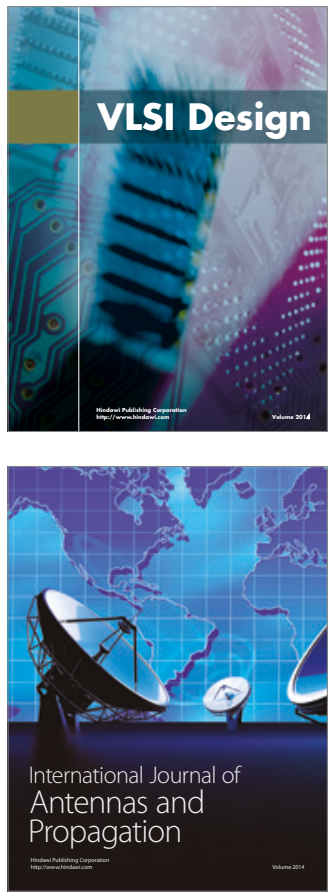

\section{Rotating}

Machinery
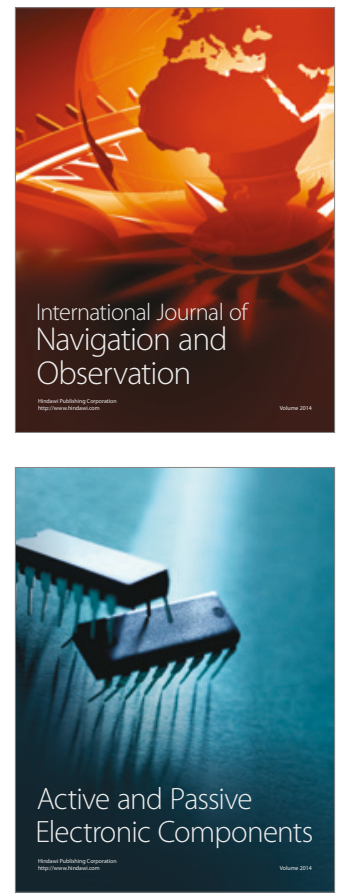
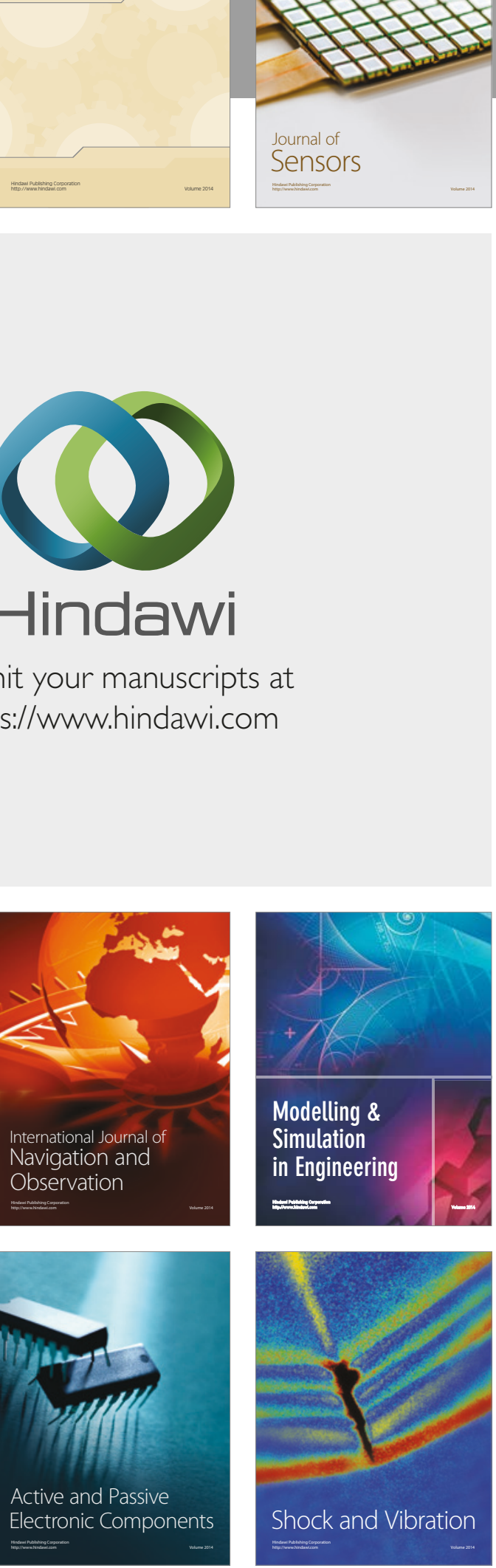
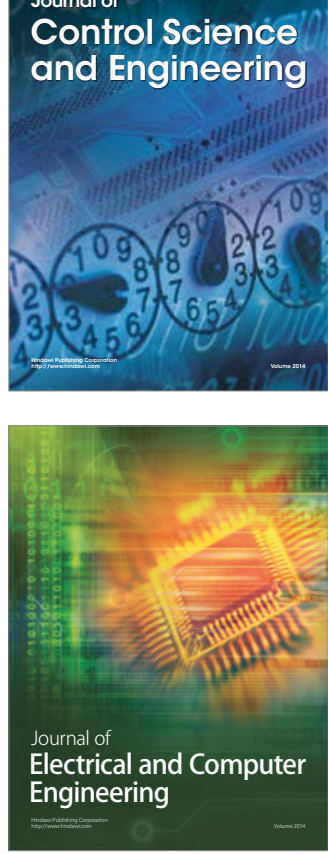

Distributed

Journal of

Control Science

and Engineering
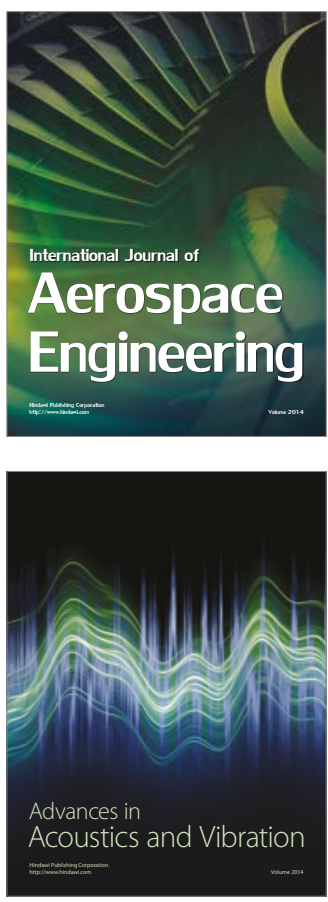

Sensor Networks 